\title{
CRITICAL CONCENTRATIONS FOR TRIPLET EXCITON TUNNELING IN BINARY NAPETHAEENE CRYSTALS: THE CASE FOR PERCOLATION
}

\author{
Eric M. MONBERG and Raoul KOPELMAN \\ Department of Chemistry, The Univeristy oj Michigen, \\ Ann Arbor, Michigan 48109, USA
}

Received 26 May 1978

Revised manuscript received 15 July 1978

\begin{abstract}
Reduced concentration eurves for triplet exciton transport are scaled by the critical concentrations in four distinct isotopic-mixed ternary sy stems. These systems with varying lifetimes, sensor concentrations and guest-host energy separations, are in excellent agreement with a two-dimensional cluster model, based on long-range percolation functions, without adjustable parameters. This supports "energy percolation".
\end{abstract}

\section{Introduction}

The concept of exciton percolation has been found to be quite useful fot the study of energy migration in isotopic mixed organic crystals $[1,2]$. It has aiso been applied to related systems, such as heterogeneous organic chromophore antenna for photosynthesis $[3,4]$. While a potential role for Anderson localization in such systems has long been realized $[1,2]$ it has been recently suggested to be the only pertinent factor $[5$, 61 , to the exclusion of exciton percolation. This conceptual confrontation has had its counterpart in earlier investigations of electron conduction systems exhibiting metal-insulator transitions [7-14].

To avoid confusion, we briefly mention four distinct, though related, kinds of percolation: classical, lattice, static and dynamic. Electrical conduction th-ough a randomly perforated metal [7] is an example of classical percolation. The concepts of cluster eigenstates, eigenvectors and absorption spectra in binary random crystals [15-21] have been related $[15,17]$ to lattice pereolation $[8,22]$ and the transition with concentration from a localized to an ex-

\footnotetext{
F Supported by NIH Grant NS08116-08.

F Present address: Western Electric, Engineering Research Center, Princeton, New Jersey 08540, USA.
}

tended nature has been termed [4] static percolation, especialiy with respect to exciton localization and delocalization and its effects on the absorption spectrum $[15,18,23]$. If one considers only near-neighbor interactions, and if the excitation lifetime is relatively long, then the exciton energy transfer is closely related $[4,21]$ to static percolation. This is found to be the case for low temperature singlet exciton transfer in naphthalene, under appropriate conditions $[21,24]$. Often, however, as time considerations play an essential role in the energy transfer, one may have the case of dynamic percolation for situations where a cut-off in interaction time is equivalent to a cut-off in interaction space [2]. The latter case is most probably related to our experimental data.

The "insulator-to-conductor" transition refers here to energy conduction via exciton tunneling. The actual mechanism has been called "trap-to-trap" exciton migration [25] and results from exciton superexchange interactions [26]. A striking aspect is the sharpness of the naphthalene $[2,27,29]$ and benzene [28] triplet exciton transfer transitions, with respect to the concentration of the "trap" (i.e., $\mathrm{C}_{10} \mathrm{H}_{8}$ or $\mathrm{C}_{6} \mathrm{H}_{6}$ ). We emphasize that, contrary to literature statements [5], we have not used "classical" percolation to describe the long range exciton tunneling $[2,4,21]$ but, starting with our earliest communication [27], we 
have emphasized the time dependent aspects of the tunneling transfer mechanism and have used the term dynamic percolation. We associate it with a cluster model of exciton migration. This model is also very different from that of an ordinary Anderson localization transition [12,14,30-32] involving diagonal inhomogeneity (in the energy), as discussed for excitons by Klafter and Jortner [5] We present new data which, in conjunction with our earlier data, are used to differentiate between the conflicting interpretations concerning the nature of the critical exciton migration transition, i.e. the "dynamic percolation" and the "Anderson localization" models.

Our conclusions, which strongly favor the dynamic cluster model ("dynamic exciton percolation") are strictly applicable only to the cited experimental systems and couditions. However, our discussion of these results should indicate when dynamic percolation and/ or Anderson localization may be of interest for excitons in general and for triplet Frenkel excitons in particular.

\section{The cluster percolation model}

A theoretical requirement for the dynamic exciton percolation model is the solution of the purely mathematical problem of "long-range-percolation" in a lattice. Little had been achieved in this direction [34] until recently, when a Monte Carlo simulation method $[2,24,35-37]$ and a semi-empirical analytical approach $[1,20]$ were developed. The key concept is the molecular cluster within which the exciton is able to move $[4,38]$.

The Monte Carlo method is used to calculate the cluster distribution as a function of the "trap" concentration and also to predict the critical site percolation concentration $C_{c}^{S}$ of the long range cluster, using the maximum in the reduced average cluster size $\boldsymbol{I}_{\mathrm{av}}$ $[24,35,36]$. In addition, we calculate the site percolation probability, $\overline{\boldsymbol{P}}_{\infty}$ or $\overline{\boldsymbol{P}}_{\max }$, which is just the probability of a guest site being included in the infinite or largest cluster, respectively. These concepts all bear upon the problem of lattice percolation [35-39].

\footnotetext{
* A specific comment on this paper is submitted separately. The "off-diagonal Anderson localization" concept as applied to excitons by Smith et al. [6] is also distinct from both dynamic and static percolation [33].
}

whether it be of the nearest neighbor or long range type. From the treatment of lattice percolation we can extract information which will be important in the formulation and testing of a dynamic percolation model. The lattice theory readily gives [36] the dependence of the cluster distribution and critical site percolation concentration $C_{\mathrm{c}}^{\mathrm{s}}$ upon the range of interaction $n$. Here $n$ is defined as the maximum number of nearest-neighbor bonds over which an interaction can occur.

We can also formulate the exciton percolation probability $P(C)$ (in the limit of supertransfer) $[21,381$ as a function of guest concentration:

$P(C)=\sum_{m}\left[1-(1-m / G)^{Z}\right] i_{m} m / G$,

where $m$ is the cluster size, $i_{m}$ the cluster frequency, $Z$ the number of sensors and $G$ the total number of guests. This is just a logical extension of the binary percolation concept into a three component system, i.e., host, guest and sensor $[4,21,38]$. Note that, formally, the sensor population is a subset of the guest population and that $i_{m}$ depencis on $n$ and thus, implicitly, on the time.

There are several approaches that can be taken to compare the dynamic percolation theory to the experimentally derived results. The first approach [2] consists of comparing the experimentally derived percolation concentration to the predicted $C_{\mathrm{c}}^{s}$ obtained by calculating an effective interaction range $n_{\text {eff }}$ from the known physical parameters. This is done independently for each experimental system. The second alternative is to use a scaling approach, based on a dimensionless guest concentration normalized with respect to an individual system's percolation concentration, to check for both model consistency and its agreement with experiment. The latter approach is entirely independent of the physical parameters, as the scaled exciton percolation curve is "universal" for a given dimensionality of interaction. Thus our test relies only on knowing the concentrations of our samples and the well established fact that the triplet naphthalene exciton interactions are predominantly "in-plane" (i.e., in the $a b$ plane) $[2,40,41]$.

\section{Results}

Fig. 1 illustrates the site percolation probability 


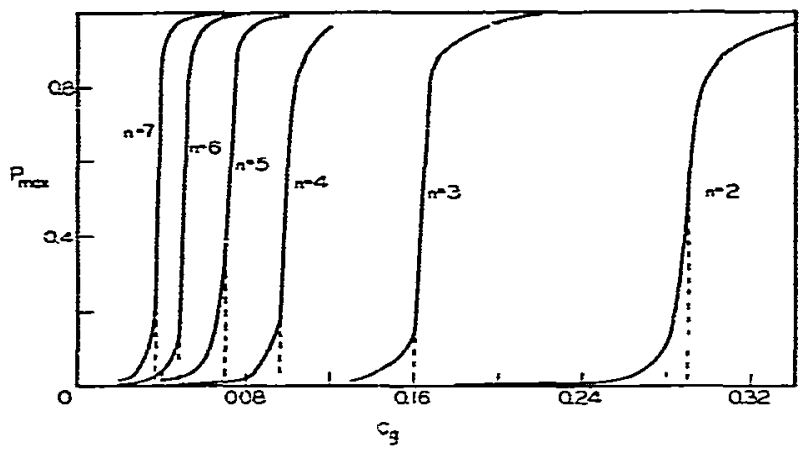

Fig- 1. Comparison of the square lattice site percolation probability, $P_{\max }$ (Drobability of the guest site being part of the largest guest cluster) for different values of the long range percolation parameter $n$, as a function of the guest concentration $C_{g}$. Here, $n$ is defined as the maximum number of nearestneighbor bonds over which an interaction (or a connection) can occur. The function $\bar{P}_{\infty}$ (Drobability of a guest being inciuded in an "infinite" cluster) is approximated by the dashed line from the $P_{\max }$ curve down to the calculated critical site concentration $C_{C}$ below which $P_{\infty}$ is zero, by definition [8]. All of the simulations for these curves were done for a square lattice of $500 \times 500$ sites. The effective coordination number is 12 for $n=2$ and 112 for $n=7$. For more details see refs. [36, 241. Note that a universal behavior has been demonstrated for all these $n$ values: When $P_{\max }$ is plotted versus the reduced concentration $\left(C-C_{\hat{C}}^{S}\right) / C_{\hat{C}}^{S}$ the six curves do practically overlap in a regular representation [53], as well as in a log-log representation [36] (giving a critical exponent [36] $\beta=0.14$ ). Note that the $n=1$ curve has been omitted as this is the well known $\bar{P}_{\infty}$ curve for the ordinary $[8,22]$ two-dimensional square lattice site peicolation problem.

[8] as a function of site concentration with the interaction range $n$ as a parameter. Fig. 2 compares the theoreticaily calculated [38] exciton percolation probability [eq. (1)] with the experimental measurements of the relative sensor/guest exciton phosphorescence $[2,24,42]$. Fig. 3 shows the same results as fig. 2 based on a scaled ("reduced") concentration. As the representation of this figure is "Liniversal", the previous experimental points are novr expected to fall on a common curve, thus giving a significantly higher measure of confidence regarding the agreement between experiment and theory (without the use of either "experimental" or "theoretical" fudge factors). The ernpirical critical concentrations $C_{\mathrm{c}}$, which scale each of the experimental series of data, have been discussed before [2] $\frac{\text { *. }}{17}$.

The quantitative agreement between the theoreti-

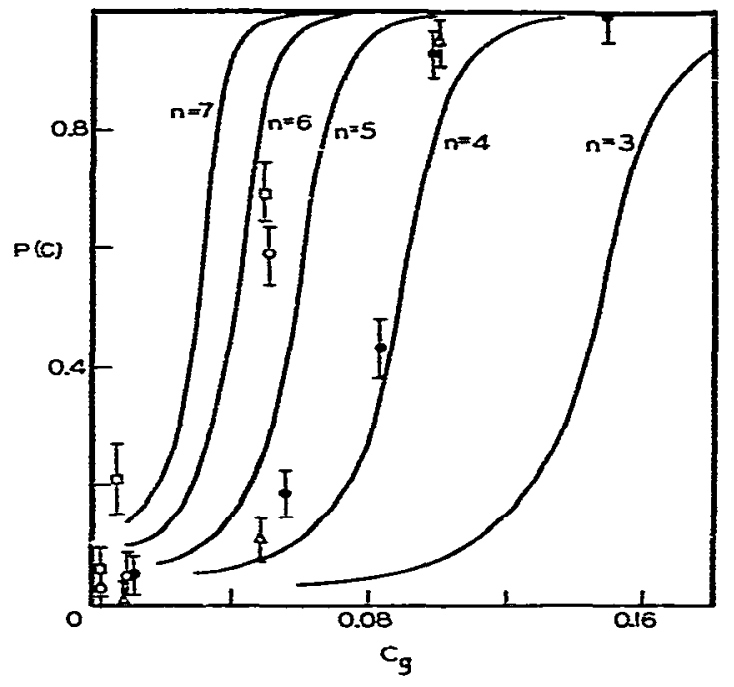

Fig. 2. The exciton percolation probability $P(C)$ as a function of guest concentration (with the interaction range $n$ as parameter for the theoretical curves). The theoretical curve is calculated from the cluster model of exciton mingration, i.e., formula (1). We have assumed a constant supertrap concentration $C_{\mathrm{S}}(=Z C / G)=5 \times 10^{-4}$. We note that this formula is based on the "supertransfer" assumption [4,381, which should be satisfactory "by definition" for dynamic percolation (i.e., time dependent connectivity). We also note that [52] $P(C) \approx$ $P_{m^{\prime}}+I_{\mathrm{av}}^{m^{\prime}} / m^{\prime}$, where $m^{\prime}=\bar{C}_{s}^{-1}=G / Z, P_{m^{\prime}}$ is the probability of a guest site being in a cluster of size $m>m^{\prime}$ and $I_{a v}^{\prime}$ is the reduced average cluster size [35] (oxcluding all the clusters where $m>m^{\prime}$ ). Again, about $C=C_{\mathrm{c}}$, and especially for $C>$ $C_{C^{\prime}}$, one has $P(C) \approx P_{m^{\prime}}$, a function that behaves quite similarly [S2] to $P_{\max }$ (fig. 1). The experimental points represent the normalized emission intensities $[2,21,24] I_{\mathrm{S}} / I_{\text {total. }}$. where $s$ designates the supertrap. These are based on some minor refinements [24] of the data $[2,271$ for the following binary systems: $\mathrm{C}_{10} \mathrm{H}_{8} / \mathrm{C}_{10} \mathrm{D}_{8}$ (solid circles), $\beta-\mathrm{D}_{1} \mathrm{C}_{10} \mathrm{H}_{7} !$ $C_{30} D_{8}$ (triangles), $\alpha-D_{1} C_{10} H_{7} / C_{10} D_{8}$ (squares) and $\alpha-D_{2} C_{10} H_{6} / C_{10} D_{8}$ (open circles). The supertrap species and concentrations, as well as the experimental conditions, are given in ref. [2]. (Notice that the respective trap depth (A) values for the four systems are $[2]: 93,86,77$ and $62 \mathrm{~cm}^{-1}$ $\left( \pm 1 \mathrm{~cm}^{-1}\right)$.).

cal exciton percolation and the experimental points, as shown in fig. 3, appears to be very satisfactory. We emphasize that the only additional parameters used in

\footnotetext{
F These have been shown elsewhere $[2,42]$ to depend on the superexchange parameters $\beta$ (pairwise interaction and $\Delta$ (trap depth) and the exciton lifetime $\tau$. The importance of the lifetime has been corroborated by spectrally resolved time evolution studies $[43,44]$.
} 


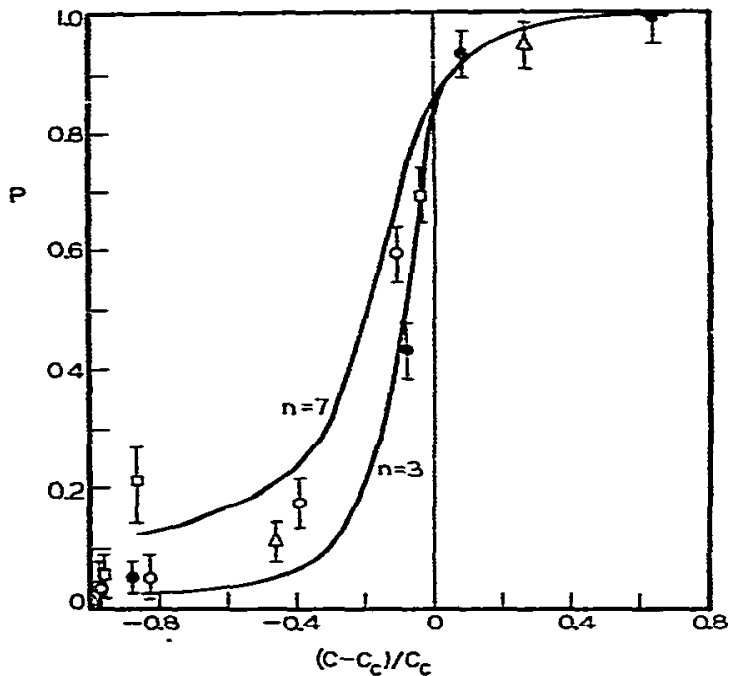

Fig. 3. Scaled exciton percolation curve. Same as in fig. 2, except that reduced guest concentrations $\left(C-C_{\mathrm{c}}\right) / C_{\mathrm{c}}$ are used. The nearly universal behavior is indicated by the small spread of the theoretical curves (the $n=4,5,6$ curves are bracketed by the $n=3$ and $n=7$ curves) and by the excellent fit of the experimental points. Note that the "spread" is caused oy the term $I_{\mathrm{av}}^{\prime \prime} / m^{\prime}$ (whose relative contribution increases with decreasing $C / C_{\mathrm{c}}$, which is a measure of the expected deviation from universality at $C<C_{C}$. The experimental critical percolation concentrations are 0.091 (solid circles), 0.088 (triangles), 0.0565 (open circles), 0.052 (squares). These were derived by first determining fractional empirical $n$ values from fig. 2 $(4.3,4.4,5.6,5.8$, respectively), and then interpolating between the appropriate $C_{C}^{\text {lines }}$ of fig. 1 . This procedure introduces an uncertainty in the reduced concentration of up to about 0.1. We note that a spread in $C_{s}$ by a factor of 2.5 is equivalent to a spread of about 0.2 in $n$.

fig. 3 are the experimental critical concentrations for each trapdepth series derived from fig. 2 . We have not used here theoretical estimates $[2,24,42]$ of $C_{c}$ based on $\beta, \Delta$ and $\tau$ (even though they are in excellent agreement with the empirical values derived below $\frac{\text { ? }}{3}$ ). The only physical assumption involved in the curves of figs. 2 and 3 is the two-dimensional topology of the triplet exciton interaction $[40,41,46-48]$. Thus we maintain that the excellent agreement of the results from the four different triplet exciton systems with the unadultered theoretical curves of fig. 3 supports percolation as a viable model for the exciton conductor-insulator transition.

$\doteqdot$ Based on a homogeneous linebroadening of $0.01 \mathrm{~cm}^{-1}$ [45].
In principle, it is always possible to prepare "bad enough" samples, where the inhomogereity $\delta$ is larger than (the pairwise interaction) $\beta$, giving an Anderson localization if $\delta$ is also the "local" inhomogeneity. However, our philosophy has been that one can learn more from the more perfect and homogeneous samples. This has been the basis for our studies on exciton interactions [49], bands [17], superexchange [26], percolation [4] and coherence [50]. We do not deny, however, that potentially interesting studies may relate to exciton inhomogeneities ${ }^{\ddagger}$.

\section{Discussion}

We have shown that our energy transfer results are fully consistent with a cluster model of exciton percolation. No adjustable parameters have been used. While this result is not necessarily [52] contradictory with Anderson localization [5], we argue in a separate "comment" against the use of Anderson localizaticn to explain our naphthalene results, and for the use of a model of phonon assisted exciton hopping from one guest cluster to another, considering the effects of exciton lifetime, sample temperature, sensor concentration and energy denominators.

Finally, we have demonstrated the power of scaling arguments and the percolation approach for the study of exciton transfer and its dimensionality. A complete theoretical and experimental study, involving critical exponents, will be given elsewhere [52].

\section{Acknowledgement}

We would like to thank James S. Newhouse for his help with the graphical presentation of our data. We also thank Professor S.D. Colson for sending us preprints of his work well ahead of publication.

\footnotetext{
* The data from a recent study on this subject [6] have been fitted with Klafter and Jortner's formulas. However, an effective $\beta$ is employed, and off-diagonal Anderson localization is invoked. Also we are in agreement with the authors [33] that the observed temperature dependence does not distinguish between Anderson localization and dynamic percolation. Or the other han 1 , it has been argued very recently [51] that the common notion of "off-diagonal disorder" does not lead to localization.
} 


\section{References}

[1] S.D. Colson, S. George, T. Keyes and V. Vaida, J. Cher:Phys. 67 (1977) 4941, and references therein.

[2] R. Kopelman, E.M. Monberg and F.W. Ochs, Chem. Phys. 19 (1977) 413, and references therein.

[3] C.E. Swenberg, R. Dominijanni and N.E. Geacintov, Photochem. Photobiol. 24 (1976) 601.

[4] R. Kopelman, in: Topics in applied physics, Vol. 15, ed. F.K. Fong (Springer, Berlin, 1976) p. 298; I. Luminescence $12 / 13$ (1976) 775; J. Phys. Chem: 80 (1976) 2191.

[5] I. Klafter and J. Jortner, Chem. Phys. Letters 49 (1977) 410.

[6] P.D. Smith, R.D. Mead and A.H. Zewail, Chem. Phys. Letters 50 (1977) 358 .

[7] B.J. Last and D.J. Thouless, Phys. Rev. Letters 27 (1971) 1719.

[8] S. Kirkpatrick, Rev. Mod. Phys. 45 (1973) 574.

[9] M. Pollak, J. Noncryst. Solids 11 (1972) 1; M. Pollak, M.L. Knotek, M.L. Kurtzman and H. Glick, Phys. Rev. Letters 30 (1973) 856.

[10] M.H. Cohen, J. Noncryst. Solids 4 (1970) 391.

11 M.H. Cohen and J. Jortner, Phys. Rev. Letters 30 (1973) 696, 699.

12] E.N. Economou and M.H. Cohen, Phys. Rev. B5 (1972) 2931.

13] N.F. Mott, Phil Mag. 29 (1974) 613; Comments Phys. 1 (1976) 203.

14] N.F. Mott, Metal-insulator transitions (Taylor and Francis, London, 1974).

15] H.-K. Hong and R. Kopelman, J. Chem. Phys. 55 (1971) 5380.

161 V.L. Broude and E.I. Rashha, Pure Appl Chem. 37 (1974) 21.

17] R. Kopelman, in: Excited states, Vol. 2, ed. E.C. Lim (Academic Press, New York, 1975) p. 33.

18] R. LeSar and R. Kopelman, Chem. Phys 29 (1978) 289.

19] J.K. Klafter and J. Jortner, VIIIth Molecular Crystal Symposium, Santa Barbara (1977).

201 S.D. Colson, S. George, T. Keyes and V. Vaida, VIIth Liolecular Crystal Symposium, Santa Rarbara (1977).

21) R. Kopelman, E.M. Monberg and F.W. Ochs, Chem. Phys. 21 (1977) 373, and references therein.

22] V.K.S. Shante and S. Kirkpatrick, Advan. Phys. 20 (1971) 325 .

23] H.-K. Hong and G.W. Robinson, J. Chem. Phys. 54 (1971) 1369.

24] E.M. Monberg, Ph.D. Thesis, University of Michigan (1977).
[25] G.C. Nieman and G.W. Robinson, J. Chem. Phys. 37 (1962) 2150; S.D. Colson and G.W. Robinson, J. Chem. Phys. 48 (1968) 2550.

[26] H.-K. Hong and R. Kopelman, Phys. Rev. Letters 25 (1970) 1030; J. Chem. Phys. S5 (1971) 724.

[27] R. Kopelman, E.M. Monberg, F.W. Ochs and P.N. Prasad, J. Chem. Phys. 62 (1975) 292.

[28] F.B. Tudron and S.D. Colson, J. Chem. Phys, 65 (1976) 4184.

[29] F.W. Ochs, Ph.D. Thesis, University of Michigan (1974).

[30] P.W. Anderson, Phys. Rev. 109 (1958) 1492.

[31] N.F. Mott, Advan. Phys. 16 (1967) 49.

[32] J.M. Ziman, J. Phy's. C2 (1969) 1230. M.H. Cohen, H. Fritzsche and S.K. Ovshinski, Phys. Rev. Letters 22 (1969) 1065.

[33] A.H. Zewail, private communication.

[34] C. Domb and M.S. Green, Phase transitions and critical phenomena (Academic Press, New York, 1972) p. 224.

[35] J. Hoshen and R. Kopelman, Phys. Rev. B14 (1976) 3438.

[36] J. Hoshen, E.M. Monberg and R. Kopelman, J. Stat. Phys, to be published.

[37] J. Hoshen and R. Kopelman, unpublished work,

[38] J. Hoshen and R. Kopelman, J. Chem. Phys. 65 (1976) 2817.

[39] J. Hoshen, P. Klymko and R. Kopelman, unpublished.

[40] D.M. Hanson, J. Chem. Phys. 52 (1970) 3409.

[41] B.J. Botter, C.J. Norhof, J. Schmidt and J.H. van der Waals, Chem. Phys. Letters 43 (1976) 210.

[42] E.M. Monberg and R. Kopelman, unpublished.

[43] P. Argyrakis, Ph.D. Thesis, The University of Michigan (1978).

[44] D. Ahlgren and R. Kopelman, unpublished.

[45] H. Port, private communication.

[46] H.C. Wolf, J. Luminessence 12/13 (1976) 33, and references therein.

[47] B.J. Botter, A.J. van Strien and J. Schmidt, Chem. Phys. Letters 49 (1977) 39.

[48] L. Altweg, M. Chaber and I. Zschokke-Granacher, Phys. Rev. B14 (1976) 1963.

[49] F.W. Ochs and R. Kopelman, J. Chem. Phys. 66 (1977) 1599.

[50] P. Argyrakis and R. Kopelman, J. Chem. Phys. 66 (1977) 3301.

[51] P.D. Antoniou and E.N. Economou, Phys. Rev. B16 (1977) 3768.

[52] R. Kopelman, E.M. Monberg and F.W. Ochs, unpublished.

[53] R. Kopelman, E.M. Monberg, J.S. Newhouse and F.W. Ochs, J. Luminescence, to be published. 